Pacific Journal of Mathematic 


\title{
FOURIER ANALYSIS AND DIFFERENTIATION OVER REAL SEPARABLE HILBERT SPACE
}

\author{
F. H. BROWNELL
}

1. Introduction. Let $l_{2}$ denote as usual the space of square summable real sequences, the prototype of real separable Hilbert space. It is well known that $l_{2}$ possesses no non-trivial, translation invariant Borel measures. However, $l_{2}$ does have infinitely many subspaces $X$, locally compact in the $l_{2}$ norm relative topology, which we may call translation spaces and for which such measures $\varphi$ exist [2]. Here the spaces $X$ are not groups under $l_{2}$ vector addition, so the notion of translation invariance must be appropriately modified. For any such $X$ we may of course use the corresponding $\varphi$ to define over $z \in l_{2}$ a Fourier transform $F$ of $f \in L_{1}(X, \mathscr{S}, \varphi)$ by

$$
F(z)=\int_{X} f(x) e^{i(z, x)} d \varphi(x) .
$$

However, in order to get the expected inverse formula, it seems necessary to be able to make $X$ into a group-roughly speaking to define a vector in $X$ corresponding to $x+y$ when this $l_{2}$ vector sum $\notin X$. This is a severe restriction on our translation spaces $X$, and the only natural ones still available seem to be essentially modifications of Jessen's infinite torus [9]. With orthogonal coordinates this is the space $X_{0}$ defined below, a modified Hilbert cube.

Since $X_{0}$ is a locally compact abelian topological group, Fourier analysis upon it becomes standard procedure. We are able to extend some standard one-variable theorems (see [1]), relating Fourier transforms and the operation of differentiation, to the situation here, which seems new. In a summary at the end we discuss the significance of these results as related to the work in functional analysis of Fréchet, Gâteaux, Lévy, Hille, Zorn, Cameron and Martin, and Friedrichs.

\section{Fourier integrals on $X_{0}$. Let}

$$
X_{0}=\left\{x \in l_{2} \mid-h_{n}<x_{n} \leq h_{n} \text { for integer } n \geq 1\right\}
$$

where the fixed sequence of extended real $h_{n}, 0<h_{n} \leq+\infty$, has

$$
\sum_{n=N+1}^{\infty} h_{n}^{2}<+\infty
$$

for some fixed integer $N \geq 0$. For simplicity we assume $h_{n}=+\infty$ for Received January 13, 1954. 
$1 \leq n \leq N$ if $N \geq 1$. Define $+^{\prime}$ addition as $l_{2}$ vector addition modulo the subgroup $I_{0}=\left\{x \in l_{2} \mid x_{n}=0\right.$ for $n \leq N, x_{n} / 2 h_{n}=m_{n}$, an integer, for $\left.n \geq N+1\right\}$. Define $P(x)$ for $x \in l_{2}$ as the unique element of $X_{0}$ in the coset $x+I_{0}$; thus clearly $x+{ }^{\prime} y=P(x+y) \in X_{0}$ for $x$ and $y \in X_{0}$. After defining the inverse $-^{\prime} x=P(-x)$ for $x \in X_{0}$, we see that $X_{0}$ becomes a group under $t^{\prime}$ and $-'$. However, the operation $t^{\prime}$ is not continuous under the metric $\|x-y\|$ defined by the $l_{2}$ norm

$$
\|x\|=\left[\sum_{n=1}^{\infty}{x_{n}}^{2}\right]^{\frac{1}{2}} .
$$

Thus, following Gelfand [5], we introduce the modified norm $\|x\|=$ $\|P(x)\|$ for $x \in l_{2}$. That $+^{\prime}$ and $-^{\prime}$ are continuous under the resulting metric $\|x-y\|$ is clear from the easily verified statements

$$
\begin{array}{r}
\left\|\left(\tilde{x}+{ }^{\prime} \tilde{y}\right)-\left(x+{ }^{\prime} y\right)\right\|=\|P(\tilde{x}-x+\tilde{y}-y)\| \leq\|P(\tilde{x}-x)+P(\tilde{y}-y)\| \\
\leq\|\tilde{x}-x\|+\|\tilde{y}-y\| \text {, and }\left\|\left(-^{\prime} y\right)-\left(-^{\prime} x\right)\right\|=\|y-x\| .
\end{array}
$$

Thus $X_{0}$ is a topological group under the metric topology of the modified norm. Note that $P(x)$ is continuous from $l_{2}$ onto $X_{0}$ under the appropriate $l_{2}$ and modified norm metrics, since

$$
\|P(x)-P(y)\|=\|P(x-y)\| \leq\|x-y\| \text {. }
$$

We can easily verify that the as yet unused condition

$$
\sum_{n=N+1}^{\infty} h_{n}{ }^{2}<+\infty
$$

is necessary and sufficient for $X_{0}$ to be locally compact under either the $l_{2}$ norm or modified norm metric topologies. Thus $X_{0}$, under the latter topology, possesses a regular Haar measure $\varphi$ defined over $\mathscr{B}$, the Borel subsets of $X_{0}$; and $\varphi$ is unique up to constant factors. Hence $\varphi$ is non-trivial and invariant under $+^{\prime}$, though, as we remarked above, this $\varphi$ could be constructed for + alone without making $X_{0}$ into a group, (see [2]). To fix $\varphi$, let

$$
V_{1}=\left\{x \in X_{0}|| x_{n} \mid<\frac{1}{2} \text { for } n \leq N\right\} ;
$$

thus $V_{1}$, being non-void and open with compact closure, must satisfy $0<\varphi\left(V_{1}\right)<+\infty$. We specify $\varphi$ uniquely by requiring $\varphi\left(V_{1}\right)=1$.

In order to get Fourier analysis on $X_{0}$ following Godement [6] or Weil [11], we need to determine the continuous characters on $X_{0}$, that is all continuous complex valued functions $\psi(x)$ on $x \in X_{0}$ with $|\psi(x)|=1$ and $\psi\left(x+{ }^{\prime} y\right)=\psi(x) \psi(y)$. Here let

$$
Z_{0}=\left\{z \in l_{2} \mid z_{n}=\frac{\pi p_{n}}{h_{n}} \text { with } p_{n} \text { an integer for } n \geq N+1\right\} \text {. }
$$


Note that since $\sum_{n=N+1}^{\infty} h_{n}{ }^{2}<+\infty$ and $z \in l_{2}$ make $h_{n} \rightarrow 0$ and $z_{n} \rightarrow 0$ as $n \rightarrow \infty$, each $z \in Z_{0}$ must have $p_{n} \equiv 0$ and thus $z_{n} \equiv 0$ for sufficiently large $n$. Let

$$
(x, y)=\sum_{n=1}^{\infty} x_{n} y_{n}
$$

denote the $l_{2}$ inner product.

LEMmA 1. The group of characters $\tilde{X}_{0}$ is iscmorphic with $Z_{0}$, each character having ihe form $\psi(x)=e^{i(z, x)}$ with $z \in Z_{0}$.

Proof. Let $\exp [i \Phi(x)]=\psi(P(x))$ for any $\psi \in \tilde{X}_{0}$, with $\Phi(0)=0$ and $\Phi(x)$ defined uniquely by requiring continuity. Thus $\Phi(x)$ is a continuous linear functional over $l_{2}$, so $\Phi(x)=(x, z)=(z, x)$ for some unique $z \in l_{2}$. For $h_{n}<+\infty$, taking $x_{j}=2 h_{n}$ if $j=n$ and $x_{j}=0$ if not, we see that $P(x)=0$. Hence $2 \pi p_{n}=\Phi(x)=(z, x)$ makes $z_{n}=\pi p_{n} / h_{n}$, so $z \in Z_{0}$.

Let $Z_{0} \subset l_{2}$ be topologized relatively from $l_{2}$. Clearly this topology is equivalent to the product of the euclidean $E_{N}$ topology with the discrete topology on the part $n>N$, where $z_{n}=\pi p_{n} / h_{n}$ and $h_{n} \rightarrow 0 . \quad Z_{0}$ so topologized forms a locally compact abelian topological group under $l_{2}$ vector addition, $\eta$ denoting its Haar measure. Clearly this topology on $Z_{0}$ is equivalent to the Hausdorff space topology with neighborhoods as finite intersections of sets of the form

$$
N_{\rho, F}\left(z_{0}\right)=\left\{z \in Z_{0}||\left(z-z_{0}, x\right) \mid<\rho \text { for } x \in F\right\},
$$

$\rho>0$ and $F$ a norm bounded subset of $X_{0}$. Equivalently on $\tilde{X}_{0}$ this topology is given by

$$
\tilde{N}_{\delta, F}\left(\psi_{0}\right)=\left\{\psi \in X_{0}\left|\psi(x)-\psi_{0}(x)\right|<\delta \text { for } x \in F\right\}
$$

Now $(X, \mathscr{B}, \varphi)$ is a $\sigma$-finite measure space, so $L_{\infty}\left(X_{0}, \mathscr{B}, \varphi\right)$ is the conjugate space of $L_{1}\left(X_{0}, \mathscr{B}, \varphi\right)$. Thus the argument of Godement, $[6, p .87]$, is valid and $Z_{0}$ is homeomorphic to $\tilde{X}_{0} \subseteq L_{\infty}\left(X_{0}, \mathscr{B}, \varphi\right)$ under the weak topology defined by $L_{1}\left(X_{0}, \mathscr{B}, \varphi\right)$.

We may normalize $\eta$ uniquely by requiring the Fourier inversion formula (2.2), which must hold as stated in Lemmas 2 and 3 following. The formulae are:

$$
F(z)=\int_{X_{0}} e^{i(z, x)} f(x) d \varphi(x)
$$




$$
f(x)=\int_{z_{0}} e^{-i(z, x)} F(z) d \eta(z) .
$$

Here we note that any $f \in L_{1}\left(X_{0}, \mathscr{B}, \varphi\right)$ has its Fourier transform $F(z)$ defined and continuous on $Z_{0}$ by $(2.1)$; and if such $F \in L_{1}\left(Z_{0}, \mathscr{B}^{\prime}, \eta\right)$, T5' being the Borel subsets of $Z_{0}$, then the right side of (2.2) also exists and is continuous. For Lemmas 2 and 3 let $/ /$ be the class of all convolutions

$$
[u * v](x)=\int_{x_{0}} u\left(x--^{\prime} y\right) v(y) d \varphi(y)
$$

of continuous functions $u(x)$ and $v(x)$ vanishing outside compact subsets of $X_{0}$. (For proof of these following well-known lemmas see [6, p. 9094]. The density of $\mathscr{C}$ in Lemma 2 follows from the regularity of $\varphi$.)

Lemma 2. $/ l$ is dense in $L_{1}\left(X_{0}, \mathscr{S}, \varphi\right)$ and $L_{2}\left(X_{0}, \mathscr{B}, \varphi\right)$, and each $f \in \mathbb{C l}$ has its Fourier transform $F \in L_{1}\left(Z_{0}\right.$, $\left.S^{\prime}, \eta\right)$ with (2.2) holding at each $x \in X_{0}$ for the inverse transformation.

Lemma 3. If $f \in L_{2}\left(X_{0}, S_{1}, \varphi\right)$, then there exists a unique Plancherel transform $F \in L_{2}\left(Z_{0}, \mathscr{C S}^{\prime}, \eta\right)$ such that every sequence $\left\{f_{k}\right\} \subseteq \mathbb{C l}$ with the $L_{2}$ norm $\left\|f-f_{k}\right\|_{2} \rightarrow 0$ also has $\left\|F-F_{k}\right\|_{2} \rightarrow 0$. Moreover, every sequence $\left\{f_{k}\right\} \subseteq \mathscr{C l}$ with $\left\|F-F_{k}\right\|_{2} \rightarrow 0$ also has $\left\|f-f_{k}\right\|_{2} \rightarrow 0$. This Plancherel transformation takes $L_{2}\left(X_{0}, \mathscr{S}, \varphi\right)$ onto $L_{2}\left(Z_{0}, \mathscr{S}^{\prime \prime}, \eta\right)$ as a Hilbert space isomorphism,

$$
\int_{X_{0}} f(x) \overline{g(x)} d \varphi(x)=\int_{z_{0}} F(z) \overline{G(z)} d \eta(z), \quad f, g \in L_{2} .
$$

In order to determine $\eta$ explicitly, let $S$ be the set of all integer valued sequences $\zeta=\left\{p_{n}\right\}$ over $n>N$ such that $p_{n}=0$ for large enough $n$ for each sequence; thus $S$ is countable. Let $z=(\omega ; \zeta)$ be defined for $\omega \in E_{N}, \zeta \in S$ by $z_{n}=\omega_{n}$ for $n \leq N$ and $z_{n}=\pi p_{n} \mid h_{n}$ for $n>N$. Letting $\chi_{A}(z)$ be the characteristic function of any $A \in \mathscr{S}^{\prime}$, with $\mu_{N}$ Lebesgue measure on $E_{N}$,

$$
\eta(A)=\left(\frac{1}{2 \pi}\right)^{N} \sum_{\zeta \in S}\left\{\int_{E_{N}} \chi_{A}(\omega ; \zeta) d \mu_{N}(\omega)\right\}=\int_{E_{N}}\left\{\sum_{\zeta \in S} \chi_{A}(\omega ; \zeta)\right\} \frac{d \mu_{N}(\omega)}{(2 \pi)^{N}}
$$

follows, by applying Lemma 3 to the Gaussian

$$
f(x)=\exp \left(-\frac{1}{2} \sum_{n=1}^{N} x_{n}^{2}\right)
$$

to determine the normalization.

3. Fourier transforms and $X_{0}$ differentiation. Here let $X_{n}$ denote $X_{0}$ with the $n$th coordinate omitted, $\varphi_{n}$ the corresponding measure over 
the $\sigma$-algebra $\mathscr{S}_{n}$ of Borel subsets of $X_{n}$, and $\mathscr{F}_{1}$ the Borel $\sigma$-algebra of $E_{1}$ if $n \leq N$, of $\left(-h_{n}, h_{n}\right)$ if $n>N$. Then [7, p. 222], we see that $\therefore=\mathscr{O}_{n} \times \mathscr{F}_{1}$ as the uncompleted product; also, using the uniqueness of Haar measure, $\varphi=\varphi_{n} \times \mu_{1}$ or $=\varphi_{n} \times\left(\mu_{1} / 2 h_{n}\right)$ according as $n \leq N$ or $>N$. Now consider $f \in L_{1}\left(X_{0}, \mathscr{S}, \varphi\right)$, let $\tilde{x}$ denote $x$ with the $n$th coordinate omitted, and define $K_{n}\left(t, x_{n}\right)=1$ if $-h_{n}<t \leq x_{n}, K\left(t, x_{n}\right)=0$ if not. Clearly $K_{n}\left(t, x_{n}\right) f\left(x_{1}, \cdots, x_{n-1}, t, x_{n+1}, \cdots\right)$ is measurable $\left(\mathscr{P}_{n} \times \mathscr{F}_{1} \times \mathscr{F}_{1}\right.$ $=\left(\mathscr{B} \times \mathscr{F}_{1}\right)$ over $\left(\tilde{x}, x_{n}, t\right) \in X_{n} \times E_{1} \times E_{1}$ if $n \leq N$, or $X_{n} \times\left(-h_{n}, h_{n}\right]$ $\times\left(-h_{n}, h_{n}\right]$ if $n>N$. Thus if we define

$$
\int f(x) d x_{n}=\int_{-\infty}^{\infty} K_{n}\left(t, x_{n}\right) f(x, t) d t,
$$

then the Fubini theorem makes $\int f(x) d x_{n} \in L_{1}\left(X_{n} \times 1, \mathscr{P}, \varphi\right)$ for any finite $x_{n}$ interval $I$.

For the following theorems we will say that $f(x)$ is $x_{n}$ absolutely continuous if for all $\tilde{x} \in X_{n}-A$, where $A$ is some set $\in \mathscr{B}_{n}$ having $\varphi_{n}(A)=0$, we have $f\left(P\left(\tilde{x}, x_{n}\right)\right)$ absolutely continuous as a function of $x_{n}$ over every finite interval of $E_{1}$.

Theorem 4. If $f \in L_{1}\left(X_{0}, \mathscr{B}, \varphi\right)$, if $f$ is $x_{n}$ absolutely continuous, and if $f_{n}^{\prime}$, the resulting $x_{n}$ first partial, is $\in L_{1}\left(X_{0}, \mathscr{P}, \varphi\right)$ also, then the (2.1) defined Fourier transforms $F_{n}$ and $F$ of $f^{\prime \prime}{ }_{n}$ and $f$ have $F_{n}(z)=$ $=-i z_{n} F(z)$ over $z \in Z_{0}$.

Proof. Consider first $h_{n}<+\infty$, so we know almost everywhere $(\varphi)$ on $X_{0}$ that

$$
f(x)=\int f_{n}^{\prime}(x) d x_{n}+f\left(P\left(\tilde{x},-h_{n}\right)\right)=\int f^{\prime}{ }_{n}(x) d x_{n}+f\left(\tilde{x}, h_{n}\right) .
$$

Now

$$
\int_{-h_{n}}^{h_{n}} e^{i z_{n} t} d t=0
$$

so

$$
F(z)=\int_{X_{n}} \int_{-h_{n}}^{h_{n}} e^{i(z, x)}\left\{\int f_{n}^{\prime}\left(x_{n}\right) d x_{n}\right\} \frac{d x_{n}}{2 h_{n}} d \varphi_{n}(\tilde{x})
$$

But

$$
\begin{aligned}
\int_{-h_{n}}^{h_{n}} e^{i z_{n} s} & \left\{\int_{-h_{n}}^{s} f_{n}^{\prime}(\tilde{x}, t) d t\right\} d s \\
& =\frac{e^{i z_{n} h_{n}}}{i z_{n}} \int_{-h_{n}}^{h_{n}} f_{n}^{\prime}(\tilde{x}, t) d t-\frac{1}{i z_{n}} \int_{-h_{n}}^{h_{n}} e^{i z_{n} s} f_{n}^{\prime}(\tilde{x}, s) d s
\end{aligned}
$$


by integrating by parts, and

$$
\int_{-h_{n}}^{h_{n}} f_{n}^{\prime}(\tilde{x}, t) d t=f\left(P\left(\tilde{x}, h_{n}\right)\right)-f\left(P\left(\tilde{x},-h_{n}\right)\right)=0 .
$$

Thus $F(z)=-\left(1 / i z_{n}\right) F_{n}(z)$ for $z_{n} \neq 0$. If $z_{n}=0$, then

$$
\int_{-h_{n}}^{h_{n}} f_{n}^{\prime}(\tilde{x}, t) d t=0
$$

makes $F_{n}(z)=0$, so $F_{n}(z)=-i z_{n} F(z)$ for all $z \in Z_{0}$.

Secondly if $h_{n}=+\infty$, we know

$$
f(x)=\int f_{n}^{\prime}(x) d x_{n}+C(\tilde{x})
$$

almost everywhere $\left(\varphi_{n}\right)$ over $\tilde{x} \in X_{n}$. Thus $f\left(\tilde{x}, x_{n}\right) \rightarrow C(\tilde{x})$ as $x_{n} \rightarrow-\infty$, so $f\left(\tilde{x}, x_{n}\right) \in L_{1}\left(E_{1}\right)$ in $x_{n}$ almost everywhere $\left(\varphi_{n}\right)$ requires $C(\tilde{x})=0$, $f(x)=\int f_{n}^{\prime}(x) d x_{n}$, and similarly $\int_{-\infty}^{\infty} f_{n}^{\prime}(\tilde{x}, t) d t=0$ almost everywhere $\left(\varphi_{n}\right)$. Thus

$$
\begin{aligned}
& \int_{-\infty}^{\infty} e^{i z_{n} s} f(\tilde{x}, s) d s=\int_{-\infty}^{\infty} e^{i z_{n} s}\left\{\int_{-\infty}^{s} f_{n}^{\prime}(\tilde{x}, t) d t\right\} d s \\
& =\lim _{a, b \rightarrow \infty}\left[\frac{e^{i z_{n} s}}{i z_{n}} \int_{-\infty}^{s} f_{n}^{\prime}(\tilde{x}, t) d t\right]_{-a}^{b}-\frac{1}{i z_{n}} \int_{-\infty}^{\infty} e^{i z_{n} s} f_{n}^{\prime}(\tilde{x}, s) d s \\
& =\frac{-1}{i z_{n}} \int_{-\infty}^{\infty} e^{i z_{n} s} f_{n}^{\prime}(\tilde{x}, s) d s, \text { so } F(z)=-\frac{1}{i z_{n}} F_{n}(z)
\end{aligned}
$$$$
\text { for } z_{n} \rightleftharpoons 0 \text {. }
$$

If $z_{n}=0$, then $\int_{-\infty}^{\infty} f_{n}{ }^{\prime}(\tilde{x}, t) d t=0$ makes $F_{n}(z)=0$, so $F_{n}(z)=-i z_{n} F(z)$ for all $z \in Z_{0}$.

For the next lemma we need to remark that $T(x ; y)=\left(x ; y-{ }^{\prime} x\right)$ is a homeomorphism of $X_{0} \times X_{0}$ into itself, and hence leaves unchanged the Borel class $\mathscr{B} \times \mathscr{B},[7, \mathrm{p} .257]$. Thus $A \in \mathscr{B}$ has $T\left(X_{0} \times A\right) \in \mathscr{B}$ $\times \mathscr{B}$, so clearly any $f(x)$ measurable $(\mathscr{B})$ has $f\left(x++^{\prime} y\right)$ measurable $(\mathscr{B} \times \mathscr{B})$. Let ${ }_{n} e \in l_{2}$ be defined by ${ }_{n} e_{k}=\delta_{n, k}$, and we then easily see, using

$$
\left\{(x ; y) \in X_{0} \times X_{0} \mid y_{k}=0 \text { for } k \neq n\right\} \in \mathscr{P} \times \mathscr{B},
$$

that such $f$ also have $f\left(x+{ }^{\prime} t_{n} e\right)$ measurable $\left(\mathscr{S}^{\prime} \times \mathscr{C}_{1}\right)$ over $x \in X_{0}$ and $t$ real.

Lemma 5. If $f \in L_{r}\left(X_{0}, \mathscr{B}, \varphi\right)$ with real $r \geq 1$, if $f$ is $x_{n}$ absolutely continuous, and if the resulting $f_{n}^{\prime} \in L_{r}\left(X_{0}, \mathscr{B}, \varphi\right)$, then defining 


$$
{ }_{n} f_{h}(x)=\frac{1}{h}\left\{f\left(x+{ }^{\prime} h_{n} e\right)-f(x)\right\}
$$

over real $h \Rightarrow 0$ yields

$$
\lim _{n \rightarrow 0}\left\|_{n} f_{h}-f_{n}^{\prime}\right\|_{r}=0 \text {. }
$$

Proof. Since $x+{ }^{\prime} h_{n} e=P\left(x+h_{n} e\right)$, we know that

$$
{ }_{n} f_{n}(x)-f_{n}^{\prime}(x)=\frac{1}{h} \int_{0}^{h}\left\{f_{n}^{\prime}\left(x+{ }^{\prime} t_{n} e\right)-f_{n}^{\prime}(x)\right\} d t
$$

almost everywhere $\left(\varphi_{n}\right)$ over $\tilde{x} \in X_{n}$. With $1 / r^{\prime}=1-1 / r$ if $r>1,1 / r^{\prime}$ replaced by 0 if $r=1$. The Schwarz-Hölder inequality thus yields

$$
\ln _{n}(x)-\left.f_{n}^{\prime}(x)|\leq| h\right|^{1 / r^{\prime}-1}\left|\int_{0}^{h}\right| f_{n}^{\prime}\left(x+{ }^{\prime} t_{n} e\right)-\left.\left.f_{n}^{\prime}(x)\right|^{r} d t\right|^{1 / r} .
$$

Then by the Fubini theorem

$$
\begin{aligned}
\left\|_{n} f_{h}-f_{n}^{\prime}\right\|_{r}^{r} & \leq \frac{1}{|h|}\left|\int_{0}^{h}\left\{\int_{x_{0}}\left|f_{n}^{\prime}\left(x+{ }^{\prime} t_{n} e\right)-f_{n}^{\prime}(x)\right|^{r} d \varphi(x)\right\} d t\right| \\
& \leq \sup _{|t| \leq \mid h_{h}}\left\|g_{t}-g\right\|_{r}^{r}
\end{aligned}
$$

where $g(x)=f_{n}^{\prime}(x) \in L_{r}$ and $g_{t}(x)=g\left(x+{ }^{\prime} t_{n} e\right)$. The functions $u(x)$, continuous on $X_{0}$ under the modified norm topology and vanishing outside compact subsets of $X_{0}$, are $L_{r}$ norm dense in $L_{r}\left(X_{0}, \mathscr{B}, \varphi\right)$ by the regularity of $\varphi$; and such $u$ have $\left\|u_{t}-u\right\|_{r} \rightarrow 0$ as $t \rightarrow 0$ by their uniform continuity. Also $\left\|g_{t}-u_{t}\right\|_{r}=\|g-u\|_{r}$ by $\varphi$ invariance, so

$$
\left\|_{n} f_{h}-f_{n}^{\prime}\right\|_{r} \leq 2\|g-u\|_{r}+\sup _{\left|t_{1} \leq h_{1}\right|}\left\|u_{t}-u\right\|_{r}
$$

and hence $\left\|f_{h}-f_{n}^{\prime}\right\|_{r} \rightarrow 0$ as $h \rightarrow 0$.

We also have the following converse for $r=2$.

Lemma 6. If $f$ and $g \in L_{2}\left(X_{0}, \mathscr{P}, \varphi\right)$ and if $\lim _{h \rightarrow 0}\left\|_{n} f_{h}-g\right\|_{2}=0$, then $f(x)=\tilde{f}(x)$ almost everywhere $(\varphi)$ for some $\tilde{f}(x)$ measurable $(\mathscr{B})$ which is $x_{n}$ absolutely continuous and has its derivative $\tilde{f}_{n}^{\prime}(x)=g(x)$ almost everywhere $(\varphi)$.

Proof.

$$
\left\|_{n} f_{h}-g\right\|_{2}^{2}=K \int_{X_{n}}\left\{\int_{-h_{n}}^{h_{n}} \ln _{h}(x)-\left.g(x)\right|^{2} d x_{n}\right\} d \varphi_{n}(\tilde{x})
$$

by the Fubini theorem, so using a Riesz-Fischer subsequence $h=t_{k} \rightarrow 0$ 
we have

$$
\lim _{t_{k} \rightarrow 0} \int_{-h_{n}}^{h_{n}}\left|f_{t_{k}}(x)-g(x)\right|^{2} d x_{n}=0
$$

for almost $\left(\varphi_{n}\right)$ all $\tilde{x} \in X_{n}$. This reduces our statement to the one real variable analogue, where the result is well known (see for example Bochner, [1, p. 131], if $\left.h_{n}=+\infty\right)$. Since we may take

$$
\tilde{f}(x)=\int_{0}^{x_{n}} g(\tilde{x}, t) d t+\tilde{f}(\tilde{x}, 0)
$$

almost everywhere $\left(\varphi_{n}\right)$ with

$$
\tilde{f}(\tilde{x}, 0)=\frac{1}{a} \int_{0}^{a}\left\{f(\tilde{x}, s)-\int_{0}^{s} g(\tilde{x}, t) d t\right\} d s
$$

for $0<a<h_{n}$, clearly $\tilde{f}(x)$ may be taken measurable $(\mathscr{B})$.

The $L_{2}$ counterpart of Theorem 4 now follows.

THEOREM 7. If $f \in L_{2}\left(X_{0}, \mathscr{B}, \varphi\right)$, if $f$ is $x_{n}$ absolutely continuous, and if the resulting $f_{n}^{\prime} \in L_{2}\left(X_{0}, \mathscr{P}, \varphi\right)$ too, then the Plancherel transforms $F$ and $F_{n}$ of $f$ and $f_{n}^{\prime}$ satisfy $F_{n}(z)=-i z_{n} F(z)$ almost everywhere $(\eta)$.

Proof. Using the Fubini theorem in (2.1) and the translation invariance of $\varphi$, we have

$$
{ }_{n} F_{h}(z)=\frac{1}{h}\left(e^{-i z_{n} h}-1\right) F(z)
$$

for the transform of ${ }_{n} f_{h}$ in case $f \in L_{1} \cap L_{2}$, and hence for all $f \in L_{2}$ by the Plancherel Lemma 3 with $L_{1} \cap L_{2}$ dense in $L_{2}$. Since

$$
\lim _{h \rightarrow 0} \frac{1}{h}\left(e^{-i z_{n} h}-1\right)=-i z_{n}
$$

and since $\left\|{ }_{n} F_{h}-F_{n}\right\|_{2} \rightarrow 0$ as $h \rightarrow 0$ by Lemma 5 and (2.3), the RieszFischer theorem yields $F_{n}(z)=-i z_{n} F(z)$ as desired.

It is easy to get an extended converse of Theorem 7 .

Theorem 8. If $f$ and $g \in L_{2}\left(X_{0}, \mathscr{B}, \varphi\right)$ and have transforms $F$ and $G$ satisfying $G(z)=\left(-i z_{n}\right)^{k} F(z)$ for integer $k>0$, then $f(x)=\tilde{f}(x)$ almost everywhere $(\varphi)$ for some $\tilde{f}(x)$ measurable $(\mathscr{S})$ such that $\tilde{f}(x)$ possesses everywhere up to $(k-1)$ st order $x_{n}$ partials which are $\in L_{2}\left(X_{0}, \mathscr{S}, \varphi\right)$, 
the $(k-1) s t \tilde{f}_{n, n \cdots, n}^{(k-1)}(x)$ is $x_{n}$ absolutely continuous, and

$$
\tilde{f}_{n, n, \ldots, n}^{(k)}(x)=g(x)
$$

almost everywhere $(\varphi)$.

Proof. From $\left(-i z_{n}\right)^{k} F(z)$ and $F(z) \in L_{2}\left(Z_{0}, \mathscr{O}^{\prime}, \eta\right)$ clearly $\left(-i z_{n}\right)^{p} F(z)$ $\in L_{2}$ also for $p=0,1, \cdots, k-1$, and by taking inverse Plancherel transforms we get ${ }_{p} g \in L_{2}\left(X_{0}, \mathscr{S}, \varphi\right)$ transforming into $\left(-i z_{n}\right)^{p} F(z)$. As we have seen before the difference quotient ${ }_{p} g_{h}$ of ${ }_{p} g$ will have the transform

$$
\frac{1}{h}\left(e^{-i z_{n} h}-1\right)\left(-i z_{n}\right)^{p} F(z)=\left\{\int_{0}^{1} e^{-i z_{n} h t} d t\right\}\left(-i z_{n}\right)^{p+1} F(z) .
$$

Since $\left\{\{\} \mid \leq 1\right.$ and \{\}$\rightarrow 1$, this transform $\rightarrow\left(-i z_{n}\right)^{p+1} F(z)$ in $L_{2}$ norm as $h \rightarrow 0$. Hence $\left\|_{p} g_{h}-{ }_{p+1} g\right\|_{2} \rightarrow 0$ as $h \rightarrow 0$ by the Plancherel lemma, and so Lemma 6 with ${ }_{0} g=f$ and ${ }_{k} g=g$ gives the result.

The following converse of Theorem 8 is considerably deeper than Theorem 7. We remark that if $f$ and $g \in L_{1}\left(X_{0}, \mathscr{S}^{\prime}, \varphi\right)$, then $f * g \in L_{1}$ also and has the Fourier transform $F(z) G(z)$, where

$$
[f * g](x)=\int_{X_{J}} f\left(x--^{\prime} y\right) q(y) d \varphi(y)
$$

exists almost everywhere $(\varphi)$. More important for us, if $f$ and $g \in L_{2}\left(X_{0}, y, \varphi\right)$, then $f * g$ is the inverse Fourier transform of $F(z) G(z) \in L_{1}\left(Z_{0}, \mathscr{P}, \eta\right)$, defined pointwise by (2.2), and hence also the inverse Plancherel transform if $F G \in L_{2}$. This follows by noting that $e^{i(z, x)} \overline{F(z)}$ is the transform of $\bar{f}\left(\overline{x-x^{\prime} y}\right)$ as a function of $y$ and by using (2.3).

Theorem 9. If $f \in L_{2}\left(X_{0}, \mathscr{B}, \varphi\right)$ and possesses everywhere up to $(k-1)$ st order $x_{n}$ partials, if $f_{n, \cdots, n}^{(k-1)}(x)$ is $x_{n}$ absolutely contiruous, and if $f_{n, \cdots, n}^{(k)}(x) \in L_{2}\left(X_{0}, \mathscr{B}, \varphi\right)$, then also $f_{n, \cdots, n}^{(p)}(x) \in L_{2}\left(X_{0}, \mathscr{B}, \varphi\right)$ for $p=1,2$, $\cdots, k$, and such $f_{n, \cdots, n}^{(p)}$ have the transforms $\left(-i z_{n}\right)^{p} F(z)$.

Proof. First we construct rather arbitrarily a smoothing transform

$$
G(z)=\exp \left(-\frac{1}{2} \sum_{j=1}^{N} \omega_{j}^{2}-\frac{1}{2} \gamma_{n} z_{n}^{2}\right) \rho(\zeta)
$$

for $z=(\omega ; \zeta)$ of $\omega \in E_{N}$ and $\zeta \in S$ using the notation of (2.4), where $\gamma_{n}=0$ if $n \leq N$ and $\gamma_{n}=1$ if $n>N$. $S$ being countable we may set $S=\left\{{ }_{k} \zeta\right\}$ and define $\rho(\zeta)$ on $S$ by setting $\rho\left({ }_{k} \zeta\right)=e^{-k}$. We see clearly from (2.4) for each integer $p \geq 0$ that $\left(-i z_{n}\right)^{p} G(z) \in L_{1} \cap L_{2} \cap L_{\infty}$ for the measure 
space $\left(Z_{0}, \mathscr{B}^{\prime}, \eta\right)$, since

$$
\left|z_{n}\right|^{p} e^{-\frac{1}{2} z_{n}^{2}}
$$

is bounded and $O\left(e^{-\left|z_{n}\right|}\right)$ as $\left|z_{n}\right| \rightarrow \infty$. Also $G(z)>0$ everywhere on $Z_{0}$, these two conditions being all we really need. Take $g$ as the unique element of $L_{2}\left(X_{0}, \mathscr{B}, \varphi\right)$ transforming into $G$, and by Theorem 8 we may take $g(x)$ to possess all order derivatives in $x_{n}$ with $g_{n, \cdots, n}^{(p)} \in L_{2}$ transforming into $\left(-i z_{n}\right)^{p} G(z)$.

Now for $h_{n}<+\infty$ and $0 \leq p \leq k$, by integrating by parts we see that

$$
\int_{-h_{n}}^{h_{n}} g_{n, \cdots, n}^{(p)}\left(x-^{\prime} y\right) f(y) d y_{n}=\int_{-h_{n}}^{h_{n}} g\left(x-^{\prime} y\right) f_{n, \cdots, n}^{(p)}(y) d y_{n}
$$

existent finite for almost $\left(\varphi_{n}\right)$ all $\tilde{y} \in X_{n}$ for each $x \in X_{0}$, using the periodicity of $g(P(x-y))$ and $f(P(y))$ at the endpoints $y_{n}= \pm h_{n}$. If $h_{n}=+\infty$ we still get the same result by a slightly different argument. Here we know $f_{n, \cdots, n}^{(k)}(x) \in L_{2}(-\infty, \infty)$ over $x_{n}$ for almost $\left(\varphi_{n}\right)$ all $\tilde{x} \in X_{n}$, so by the Schwarz inequality follows

$$
f_{n, \cdots, n}^{(k-1)}(x)=O\left(\left|x_{n}\right|\right)
$$

as $\left|x_{n}\right| \rightarrow \infty$ for such $\tilde{x}$. Thus by further integration

$$
f_{n, \cdots, n}^{(p)}(x)=O\left(\left|x_{n}\right|^{k}\right)
$$

as $\left|x_{n}\right| \rightarrow \infty$ for such $\tilde{x}, 0 \leq p \leq k-1$. Now clearly

$$
g(x)=e^{-\frac{1}{2} x_{n}^{2}} g_{1}(\tilde{x}),
$$

so

$$
g_{n, \cdots, n}^{(p)}(x)=O\left(e^{-\left|x_{n}\right|}\right)
$$

as $\left|x_{n}\right| \rightarrow \infty$. These two estimates are enough to make the endpoint terms vanish in integration by parts, so

$$
\int_{-\infty}^{\infty} g_{n, \cdots, n}^{(p)}\left(x-{ }^{\prime} y\right) f(y) d y_{n}=\int_{-\infty}^{\infty} g\left(x-^{\prime} y\right) f_{n, \cdots, n}^{(p)}(y) d y_{n}
$$

Thus with $K=1$ or $1 / 2 h_{n}$ we have

$$
\left[g_{n, \cdots, n}^{(p)} * f\right](x)=K \int_{X_{n}} \int_{-h_{n}}^{h_{n}} g\left(x-{ }^{\prime} y\right) f_{n, \cdots, n}^{(p)}(y) d y_{n} d \varphi_{n}(\tilde{y})
$$

existent finite in the order written for $0 \leq p \leq k$ and all $x \in X_{0}$.

Now for $p=k$ we are given $f_{n, \cdots, n}^{(k)} \in L_{2}$, so the Schwarz inequality shows $g\left(x-x^{\prime} y\right) f_{n, \cdots, n}^{(k)}(y)$ to be $\in L_{1}$. Thus by the Fubini theorem 


$$
\left[g_{n, \cdots, n}^{(k)} * f\right](x)=\left[g * f_{n, \cdots, n}^{(k)}\right](x)
$$

at all $x \in X_{0}$. By our remarks preceding this theorem, since $\left(-i z_{n}\right)^{k} G(z)$ and $G(z) \in L_{\infty}$ make $\left(-i z_{n}\right)^{k} G(z) F(z)$ and $G(z) F_{k}(z) \in L_{2}$, for the Plancherel transforms we have $\left[\left(-i z_{n}\right)^{k} G(z)\right] F(z)=G(z) F_{k}(z)$. Thus since $G(z)>0$ everywhere, $F_{k}(z)=\left(-i z_{n}\right)^{k} F(z)$ with $F_{k} \in L_{2}$ the transform of $f_{n, \cdots, n}^{(k)} \in L_{2}$. Thus Theorem 8 gives the result.

THEOREM 10. If $f$ and $g \in L_{2}\left(X_{0}, \mathscr{B}, \varphi\right)$ and if their transforms $F$ and $G$ satisfy

$$
G(z)=-\left(\sum_{n=1}^{\infty} z_{n}^{2}\right) F(z),
$$

then there exists a sequence of functions ${ }_{n} f(x)$ measurable $(\mathscr{B})$ such that ${ }_{n} f(x)=f(x)$ almost everywhere $(\varphi),{ }_{n} f(x)$ is $x_{n}$ absolutely continuous as well as its everywhere existent first $x_{n}$ derivative ${ }_{n} f_{n}^{\prime}(x),{ }_{n} f_{n}^{\prime}$ and ${ }_{n} f_{n n}^{\prime \prime} \in L_{2}\left(X_{r}, \mathscr{B}, \varphi\right)$, and $\sum_{n=1}^{M}{ }_{n} f_{n n}^{\prime \prime}$ converges in $L_{2}$ norm to $g$ as $M \rightarrow \infty$.

Proof. Let $g_{n} \in I_{2}\left(X_{0}, \mathscr{B}, \varphi\right)$ be defined uniquely by requiring $G_{n}(z)=-z_{n}^{2} F(z)$, since $\left|z_{n}^{2} F(z)\right| \leq|G(z)|$ makes $z_{n}^{2} F(z) \in L_{2}\left(Z_{0}, \mathscr{B}^{\prime}, \eta\right)$. Now $\sum_{n=1}^{\infty} z_{n}^{2}$ is actually a finite sum for each $z \in Z_{0}$, and also

$$
\left|\sum_{n=1}^{M} z_{n}^{2}\right||F(z)| \leq|G(z)| \in I_{2}
$$

so by dominated convergence $\sum_{n=1}^{M} G_{n}(z) \rightarrow G(z)$ in $L_{2}$ norm as $M \rightarrow \infty$, and hence also $\sum_{n=1}^{M} g_{n} \rightarrow g$ in $L_{2}$ norm. Finally Theorem 8 for each $n$ gives the desired ${ }_{n} f(x)=f(x)$ almost everywhere $(\varphi),{ }_{n} f_{n}^{\prime}$ and ${ }_{n} f_{n n}^{\prime \prime} \in L_{n}$, and ${ }_{n} f_{n n}^{\prime \prime}(x)=g_{n}(x)$ almost everywhere $(\varphi)$ as desired.

THEOREM 11. Let $f$ and $g \in L_{2}\left(X_{0}, \mathscr{B}, \varphi\right)$ and let a sequence of functions ${ }_{n} f(x)$ measurable $(\mathscr{B})$ satisfy the conditions: ${ }_{n} f(x)=f(x)$ almost everywhere $(\varphi)$; ${ }_{n} f$ everywhere possesses a first $x_{n}$ partial ${ }_{n} f_{n}^{\prime}$ which is $x_{n}$ absolutely continuous ; ${ }_{n} f_{n n}^{\prime \prime} \in L_{2}\left(X_{0}, \mathscr{P}, \varphi\right)$; and $\sum_{n=1}^{M}{ }_{n} f_{n n}^{\prime \prime} \rightarrow g$ in $L_{2}\left(X_{0}, \mathscr{B}, \varphi\right)$ norm as $M \rightarrow \infty$. Then the transforms $F$ and $G$ satisfy

$$
G(z)=-\left(\sum_{n=1}^{\infty} z_{n}^{2}\right) F(z)
$$

almost everywhere $(\eta)$.

Proof. By Theorem 9 we also have ${ }_{n} f_{n}^{\prime} \in L_{2}$ and ${ }_{n} f_{n n}^{\prime \prime}$ has the transform $G_{n}(z)=-z_{n}^{2} F(z)$. From $\sum_{n=1}^{M}{ }_{n} f_{n n}^{\prime \prime} \rightarrow g$ in $L_{2}$ we thus know 
$\sum_{n=1}^{M} G_{n} \rightarrow G$ in $L_{2}$ norm as $M \rightarrow \infty$, where

$$
\sum_{n=1}^{M} G_{n}(z)=-\left(\sum_{n=1}^{M} z_{n}^{2}\right) F(z)
$$

Since $\sum_{n=1}^{\infty} z_{n}^{2}$ is actually a finite sum at each $z \in Z_{0}$, Riesz-Fischer subsequences yield

$$
G(z)=-\left(\sum_{n=1}^{\infty} z_{n}^{2}\right) F(z)
$$

as desired.

4. Significance of results. The main results of this paper are Theorems 7 through 11 relating Fourier transforms over $X_{0}$, a modification of the Hilbert cube, to the operations of differentiation in an $L_{2}$ sense. It is clear that Theorems 10 and 11 allow one to use Fourier transforms to define a generalized Laplace differential operator for scalar functions on $X_{0}$. This definition is in a global $L_{2}$ sense, which gives a pointwise definition only by using Riesz-Fischer subsequences. The ideas of pointwise infinite dimensional derivatives seem to go back to Fréchet and Gâteaux. Hille [8, pp. 71-90], Zorn [12], and others have developed a notion of analyticity from similar complex differentiability on complex Banach spaces.

To be precise, for real $l_{\text {, }}$ consider a real valued function $f(x)$ over $x \in l_{2}$ and define the gradient $\nabla f(x)=y$ at each $x$ such that there exists $y \in l_{\text {, }}$ having over $u \in l_{\text {, }}$

$$
\lim _{\|u\| \rightarrow 0}\|u\|^{-1}|f(x+u)-f(x)-(u, y)|=0,
$$

such $y$ being clearly unique. This is a Fréchet type definition. If we let $\left\{w_{n}\right\}$ be a complete orthonormal system in $l_{y}$, we have where $\nabla f(x)$ exists that

$$
\left(w_{n}, \nabla f(x)\right)=\left[\frac{d}{d \lambda} f\left(x+\lambda w_{n}\right)\right]_{\lambda=0} .
$$

This equation could also serve as a Gâteaux type definition of $\nabla f(x)$, possibly depending on $\left\{w_{n}\right\}$, wherever the squares of the right hand terms are summable. For the divergence, if $T(x) \in l_{2}$ for each $x \in l_{2}$, we may formulae the Gâteaux type definition

$$
(\nabla, T(x))=\sum_{n=1}^{\infty}\left(w_{n}, \nabla \psi_{n}(x)\right) \text { for } \psi_{n}(x)=\left(T(x), w_{n}\right),
$$

which is independent of the choice of base $\left\{w_{n}\right\}$ if 


$$
\begin{gathered}
\sum_{n=1}^{\infty}\left\|\nabla \psi_{n}(x)\right\|<+\infty \quad \text { and } \\
0=\lim _{\|x\| \rightarrow 0}\|u\|^{-1}\left[\sum_{n=1}^{\infty}\left|\psi_{n}(x+u)-\psi_{n}(x)-\left(u, \nabla \psi_{n}(x)\right)\right|^{2}\right]^{\frac{1}{2}} .
\end{gathered}
$$

Finally we can define the Laplacian $\nabla^{2} f(x)=(\nabla, \nabla f(x))$, so that

$$
(\nabla, \nabla f(x))=\sum_{n=1}^{\infty}\left[\frac{d^{2}}{d \lambda^{2}} f\left(x+\lambda w_{n}\right)\right]_{\lambda=0}
$$

shows this definition to agree pointwise with the expression in Theorems 10 and $11, \sum_{n=1}^{\infty} f_{n n}^{\prime \prime}(x)$.

Lévy has also constructed a vector analysis for Hilbert space, though he is led to define

$$
\lim _{M \rightarrow \infty} \frac{1}{M}\left\{\sum_{n=1}^{M} f_{n n}^{\prime \prime}(x)\right\}
$$

as the Laplacian, [5, p. 248]. He differs more seriously from our approach by using a development of mean values of functions instead of integration with respect to a non-trivial, translation invariant measure. Thus he has no need to reduce $l_{2}$ to $X_{0}$, though naturally his theory of mean values must pay for this by certain anomalous features. Cameron and Martin have also done a great deal of functional analysis in terms of Wiener measure on the continuous functions ([3] and others), but since this is not translation invariant, it has little contact with our work.

It seems that our results relating Fourier transforms and differentiation over real Hilbert space may be useful in a mathematical formulation of quantum radiation theory, just as finite dimensional differential operators are very conveniently defined self-adjointly in terms of Fourier transforms. Friedrichs has discussed such problems and is led to still a different method of integration over Hilbert space, [4, p. 60]. However, the set functions induced by his method are not $\sigma$-additive and apparently not translation invariant either.

\section{REFERENCES}

1. S. Bochner and K. Chandrasekharan, Fourier transforms, Ann. Math. Studies, 19 (1949).

2. F. H. Brownell, Translation invariant measure over separable Hilkert space and other translation spaces, Pacific J. Math., 2 (1952), 531-553.

3. R. H. Cameron and W. T. Martin, Wiener integrals under linear transformations, Trans. Amer. Math. Soc., 58 (1945), 184.

4. K. O. Friedrichs, Quantum theory of fields, Comm. Pure Appl. Math., 4 (1951) and 5 (1952). 
5. I. Gelfand, Normierte Ringe, Rec. Math., N. S. 9 [51], (1941), 3-24.

6. R. Godement and H. Cartan, Analyse harmonique, Ann. Sci. Ecole Norm. Sup., 64 (1947), 79.

7. P. R. Halmos, Measure theory, 1950, N. Y.

8. E. Hille, Functional analysis and semi-groups, Amer. Math. Soc. Colloquium Publication, 31, 1948, N. Y.

9. B. Jessen, Theory of integration in a space of infinite dimension, Acta Math., 63 (1934), 249-323.

10. P. Lévy, Problemes concrets d'analyse fonctionelle, 1951, Paris.

11. A. Weil, L'Integration dans les groupes topologiques et ses ápplications, Actualites Sci. Ind., 1940, Paris.

12. M. Zorn, Derivatives and Fréchet differentials, Bull. Ame̊r. Math. Soc., 52 (1946), 133.

\section{UNIVERSITY OF WASHINGTON}




\section{PACIFIC JOURNAL OF MATHEMATICS}

\section{EDITORS}

\author{
H. L. Royden \\ Stanford University \\ Stanford, California \\ E. Hewite \\ University of Washington \\ Seattle 5 , Washington
}

\author{
R. P. Dilworth
}

California Institute of Technology Pasadena 4, California

\section{A. HorN*}

University of California

Los Angeles 24, California

\section{ASSOCIATE EDITORS}

\author{
H. BUSEMANN \\ HERBERT FEDERER \\ MARSHALL HALL
}

\author{
P. R. HALMOS \\ HEINZ HOPF \\ ALFRED HORN
}

\author{
R. D. JAMES \\ BORGE JESSEN \\ PAUL LÉVY
}

GEORGE PÓLYA

J. J. STOKER

KOSAKU YOSIDA

\section{SPONSORS}

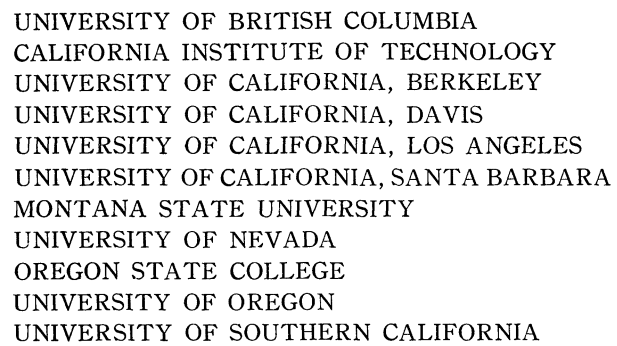

UNIVERSITY OF BRITISH COLUMBIA

CALIFORNIA INSTITUTE OF TECHNOLOGY

UNIVERSITY OF CALIFORNIA, BERKELEY

UNIVERSITY OF CALIFORNIA, DAVIS

UNIVERSITY OF CALIFORNIA, LOS ANGELES

UNIVERSITY OF CALIFORNIA, SANTA BARBARA

MONTANA STATE UNIVERSITY

UNIVERSITY OF NEVADA

OREGON STATE COLLEGE

UNIVERSITY OF OREGON

UNIVERSITY OF SOUTHERN CALIFORNIA

\author{
STANFORD RESEARCH INSTITUTE \\ STANFORD UNIVERSITY \\ UNIVERSITY OF UTAH \\ WASHINGTON STATE COLLEGE \\ UNIVERSITY OF WASHINGTON
}

AMERICAN MATHEMATICAL SOCIETY

HUGHES AIRCRAFT COMPANY SHELL DEVELOPMENT COMPANY

Mathematical papers intended for publication in the Pacific Journal of Mathematics should be typewritten (double spaced), and the author should keep a complete copy. Manuscripts may be sent to any of the editors. Manuscripts intended for the outgoing editors should be sent to their successors. All other communications to the editors should be addressed to the managing editor, Alfred Horn at the University of California Los Angeles 24, California.

50 reprints of each article are furnished free of charge; additional copies may be obtained at cost in multiples of 50 .

The Pacific Journal of Mathematics is published quarterly, in March, June, September, and December. The price per volume (4 numbers) is $\$ 12.00$; single issues, $\$ 3.50$; back numbers (Volumes $1,2,3)$ are available at $\$ 2.50$ per copy. Special price to individual faculty members of supporting institutions and to individual members of the American Mathematical Society: $\$ 4.00$ per volume; single issues, $\$ 1.25$.

Subscriptions, orders for back numbers, and changes of address should be sent to the publishers, University of California Press, Berkeley 4, California.

Printed at Kokusai Bunken Insatsusha (International Academic Printing Co., Ltd.) No. 10 1-chome Fujimi-cho, Chiyoda-ku, Tokyo, Japan.

* During the absence of E. G. Straus. 


\section{Pacific Journal of Mathematics}

\section{Vol. 5, No. $5 \quad$ BadMonth, 1955}

Henry A. Antosiewicz, A theorem on alternatives for pairs of matrice . . . . . 641

F. V. Atkinson, On second-order non-linear oscillation ............... 643

Frank Herbert Brownell, III, Fourier analysis and differentiation over real separable Hilbert spac .................................. 649

Richard Eliot Chamberlin, Remark on the averages of real function ...... 663

Philip J. Davis, On a problem in the theory of mechanical quadrature ... . . 669

Douglas Derry, On closed differentiable curves of order $n$ in $n$-spac ...... 675

Edwin E. Floyd, Boolean algebras with pathological order topologie ... . . 687

George E. Forsythe, Asymptotic lower bounds for the fundamental frequency of convex membrane ................................. 691

Israel Halperin, On the Darboux propert ................... 703

Theodore Edward Harris, On chains of infinite orde .............. 707

Peter K. Henrici, On certain series expansions involving Whittaker functions

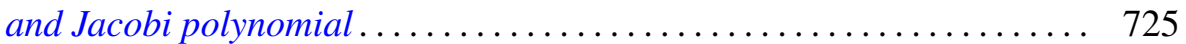

John G. Herriot, The solution of Cauchy's problem for a third-order linear hyperoblic differential equation by means of Riesz integral ......... 745

Jack Indritz, Applications of the Rayleigh Ritz method to variational

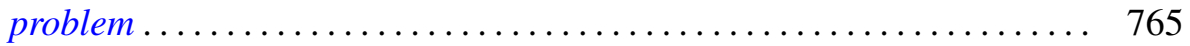

E. E. Jones, The flexure of a non-uniform bea ................. 799

Hukukane Nikaidô and Kazuo Isoda, Note on non-cooperative convex game.

Raymond Moos Redheffer and W. Wasow, On the convergence of asymptotic solutions of linear differential equation . . .

S. E. Warschawski, On a theorem of L. Lichtenstei ...........

Philip Wolfe, The strict determinateness of certain infinite game... 